\title{
Salt-finger convection generated by lateral heating of a solute gradient
}

\author{
By C. F. CHEN ${ }^{1}$ AND FALIN CHEN ${ }^{2}$ \\ ${ }^{1}$ Department of Aerospace and Mechanical Engineering, The University of Arizona, \\ Tucson, AZ 85721, USA \\ ${ }^{2}$ Institute of Applied Mechanics, National Taiwan University, Taipei, Taiwan 10764 , \\ Republic of China
}

(Received 2 July 1996 and in revised form 14 April 1997)

When a tank of fluid with a solute gradient is subjected to lateral heating, a series of horizontal convection cells is generated when the critical condition is exceeded. This phenomenon has been observed experimentally and simulated by two-dimensional numerical schemes by a number of previous investigators. In each of the convection cells, relatively warm and solute-rich fluid flows from the hot to the cold wall along the top of the cell while the return of the relatively cool and solute-poor fluid is along the bottom of the cell. This situation is conducive to the onset of salt fingers. We recently performed a series of such experiments with salt-water and ethanol-water solutions. By using flow visualization techniques, salt fingers in longitudinal rolls typical of those occurring in shear flows were observed. Salt fingers were observed as soon as convection was initiated, and they advanced with the convection front. Experiments with an ethanol-water solution showed that salt fingers can be generated by flows driven by a surface tension gradient and that the effect of solute concentration on surface tension plays an important role in the process.

\section{Introduction}

When a tank of fluid with a solute gradient is subjected to lateral heating, a series of horizontal convection cells is generated when the critical condition is exceeded. Thorpe, Hutt \& Soulsby (1969) used a linear stability analysis to predict that, if the lateral temperature gradient is established gradually, the critical condition is

$$
R_{T}=0.059\left(-R_{S}\right)^{5 / 6}
$$

where the Rayleigh numbers are defined as $R_{T}=g \alpha \Delta T D^{3} / \kappa \nu$ and $R_{S}=g \beta(\partial S /$ $\partial z) D^{4} / \kappa_{S} \nu$. In these expressions, $g$ is the gravitational acceleration, $\alpha$ and $\beta$ are the coefficients of expansion due to heat and solute, respectively, $\Delta T$ is the temperature difference across the tank width $D, \kappa$ and $\kappa_{S}$ are the diffusivities of heat and solute, and $v$ is the kinematic viscosity. This result was confirmed by experiments conducted at large values of $R_{S}$. Hart (1971) considered the stability problem, taking into account the boundary layer flow neglected by Thorpe et al. He showed that the instability is shear dominated at low values of $-R_{S}$ and double-diffusive dominated at high values of $-R_{S}$ and the critical condition given by (1) becomes asymptotically correct as $-R_{S} \rightarrow \infty$. In a subsequent finite-amplitude analysis of the problem, Hart (1973) showed that if $L e=\kappa / \kappa_{S}>\left(-R_{S}\right)^{1 / 6}$, the onset of instability is through a subcritical bifurcation and the streamline pattern may be considerably different from that 
predicted by the linear theory. The nonlinear phenomenon was studied in more detail numerically using a Galerkin method by Thangam, Zebib \& Chen (1982) for both vertical and inclined geometries. Their results show that the counter-rotating vortices at onset as predicted by the linear stability theory merge into vortices with twice the wavelength, rotating in the same direction, as the critical Rayleigh number is exceeded.

Consider the case in which $\Delta T$ across the tank is applied by setting $\pm \Delta T / 2$ at the hot and cold walls, respectively, and the tank width is much larger than the thermal diffusion length, $D \gg(\kappa t)^{1 / 2}$, where $t$ denotes the time of the onset of instabilities. We shall refer to this case as the 'impulsively applied $\Delta T$ case', even though the test tank has a non-zero time constant. Chen, Briggs \& Wirtz (1971) showed that the relevant length scale of the problem is $h$, the height of the rise of a heated fluid element in the given density gradient due to the solute distribution

$$
h=\frac{\alpha(\Delta T / 2)}{\beta(-\mathrm{d} S / \partial z)} .
$$

Through a series of experiments, Chen et al. determined the critical Rayleigh number, $\widetilde{R}$, based on $h$ to be

$$
\tilde{R}=\frac{g \alpha(\Delta T / 2) h^{3}}{\kappa \nu}=15000 \pm 2500 .
$$

Further, they found that the average wavelength of the convection cells in a range of Rayleigh numbers, $1.4 \times 10^{4}<\widetilde{R}<5.4 \times 10^{4}$, varied from $0.6 h$ to $0.97 \mathrm{~h}$. Huppert \& Turner (1980), in an experiment with a melting ice block in a salt gradient, found that, for $\widetilde{R} \sim 5-10 \times 10^{5}$, the wavelength became smaller, $0.62 h$. Huppert, Kerr \& Hallworth (1984) found the result to be valid for a number of aqueous solutions with a wide range in the values of Prandtl number $P r$ and Le. Kerr (1989) performed a linear stability analysis of this problem and showed that the stability is governed by a simple nondimensional parameter, $Q$, which is related to a Rayleigh number incorporating both the length scale $h$ and the thermal diffusion length scale. His results show that the critical wavelength is essentially $h$, and this predictions of the critical conditions are in general agreement with the experimental results of Chen et al. (1971) and in good agreement with those of Tanny \& Tsinober (1988). Later, Kerr (1990) carried out a weakly nonlinear analysis and found that the bifurcation to instability is subcritical, similar to the gradual heating case.

There have been a number of numerical simulations of the impulsively heated case. All of these are based on the assumption of two-dimensional motion. Wirtz, Briggs \& Chen (1972) used a finite difference method to demonstrate the onset of cellular convection successively at lower Rayleigh numbers and simultaneously at higher Rayleigh numbers, as found in the experiments of Chen et al. (1971). Heinrich (1984) used a finite element method and the results compared well with the experiments of Chen et al. (1971). More recently, Lee \& Hyun (1991) used a finite difference method to simulate the experiments of Chen et al. (1971), Wirtz et al. (1972), and Huppert \& Turner (1980) with good results. Their results further confirm that $h$ is a good estimate for the thickness of the convection layers. More recent efforts include those by Wright \& Shyy (1996), who studied the effect of constant heat flux rather than constanttemperature boundary, and Chen \& Liou (1997), who considered the effect of the inclination of the test tank.

The flow in the well-developed convection cells was suspected to be a threedimensional one because of the possibility of the existence of salt fingers (Thorpe et al. 
1969, Wirtz et al. 1972). Thorpe et al. stated that "the mechanism of mixing at the cell boundaries was not clear, but in the well-developed layers there frequently appeared to be narrow pencils of fluid, when coloured by dye, which looked very similar to salt fingers' (p. 394). Wirtz et al. measured the vertical distributions of salinity and temperature along the centre of the tank and found that, in general, both are higher (lower) near the top (bottom) cell boundary. Because salt fingers provide efficient transport of both salinity and heat, their presence can greatly enhance the mixing within each convection cell, influencing the onset, maintenance, and eventual merging of the cells. In order to understand these various processes and to formulate theoretical predictions by either nonlinear analysis or numerical simulation, better knowledge of the fingering phenomenon occurring in the convection cells is needed. We have undertaken such an experimental study, and the results are reported herein.

\section{Experiments}

The experiments were carried out in a tank $5 \mathrm{~cm}$ wide $\times 9.5 \mathrm{~cm}$ high $\times 9.5 \mathrm{~cm}$ long. The two sidewalls were made of chrome-plated copper, with passages provided for circulating fluid from two constant-temperature baths. The two endwalls were made of Plexiglas, and the bottom of Bakelite. A four-electrode conductivity probe and a thermocouple were mounted on a vertical traverse mechanism located at the centre of the tank to measure the concentration and temperature of the salt solution. The conductivity probe was temperature compensated using the polynomial recommended by Head (1983).

Two solutions were used to obtain the initial stratification. One was a salt solution with concentrations varying from $2 \%$ salt $\left(\rho=1.014 \mathrm{~g} \mathrm{~cm}^{-3}\right)$ to $5 \%$ salt $(\rho=1.036 \mathrm{~g}$ $\mathrm{cm}^{-3}$ ) with $\Delta \rho / \rho=0.0215$. We selected this relatively small density gradient in order to obtain a reasonable critical $\Delta T$ across the tank for the onset of double-diffusive layered convection. The critical $\Delta T$ is $8.4^{\circ} \mathrm{C}$ for an impulsively applied $\Delta T$ (Chen et al. 1971) and $6.5^{\circ} \mathrm{C}$ for a gradually increased $\Delta T$ (Thorpe et al. 1969). The other is an ethanol-water solution used to examine whether capillary motion along the free surface would generate salf-finger convection. We chose an ethanol-water solution for two reasons. One is that contamination of the free surface by impurities is not likely to occur, and the other is that the solution is a Soret fluid, which can be stratified by imposing a temperature gradient, a property useful in Space experiments. Of course, the effect of Soret diffusion on the stability of the double-diffusive system needs to be taken into account properly (Knobloch 1980). The volumetric expansion coefficients of rich ethanol-water solutions are approximately five times that for pure water. In order to achieve approximately the same critical $\Delta T$ for the salt solution, we selected a concentration variation from $100 \%$ ethanol $\left(\rho=0.791 \mathrm{~g} \mathrm{~cm}^{-4}\right)$ to $64 \%$ ethanol $\left(\rho=0.883 \mathrm{~g} \mathrm{~cm}^{-1}\right)$ with $\Delta \rho / \rho=0.1099$, almost 5 times the value for the salt solution. The corresponding critical $\Delta T$ are $8.6^{\circ} \mathrm{C}$ (Chen et al. 1971) and $6.2^{\circ} \mathrm{C}$ (Thorpe et al. 1969).

It is noted here that, in the neighbourhood of the free surface where the capillary flow is active, the solution is nearly $100 \%$ ethanol with the Soret coefficient approaching zero. The effect of Soret diffusion on salt-finger convection is likely to be negligible. In all the experiments, the desired $\Delta T$ was achieved by increasing (decreasing) $\Delta T / 2$ at the hot (cold) wall simultaneously.

Flow visualization was carried out either by shadowgraph or by particle trace. For the latter, aluminium powder was added to the prepared solutions for each of the layers prior to filling the tank. A $20 \mathrm{~mW}$ He-Ne laser with a cylindrical lens was used to 
provide a horizontal sheet of light through the liquid in the tank. A CCD video camera was mounted vertically above the tank, and the image of the particle motion was viewed on a monitor and simultaneously recorded by a time-lapse VCR. The test tank was mounted on a platform capable of vertical motion. In this manner, particle traces could be viewed at any horizontal plane within the $10 \mathrm{~cm}$ height of the test tank.

Twenty-one layers, each containing $21.5 \mathrm{~cm}^{3}$ of solutions of decreasing density, were successively introduced into the tank to obtain the initial stratification. One hour after the completion of filling with the salt solution, concentration measurements made every $2.5 \mathrm{~mm}$ vertically along the centre of the tank showed a linear distribution with height, except near the top and bottom boundaries. For the ethanol-water solution, the diffusivity varied nonlinearly with the concentration from $0.41 \times 10^{-5} \mathrm{~cm}^{-2} \mathrm{~s}^{-1}$ at $64 \%$ ethanol to $1.15 \times 10^{-5} \mathrm{~cm}^{2} \mathrm{~s}^{-1}$ at $100 \%$ ethanol (Landolt \& Bornstein 1969). Since we have no means of measuring the concentration of ethanol, the diffusion process was simulated by a one-dimensional calculation based on the diffusion equation with diffusivity a given function of concentration. The concentration distribution at $1 \mathrm{~h}$ after the start of the diffusion process was essentially linear with height, with nondiffusive effects near the top and bottom. With these results in mind, the test procedure followed was to begin all experiments $1 \mathrm{~h}$ after completion of the filling process.

For experiments with a free surface, the top boundary was not used. This resulted in a tank with height $=10 \mathrm{~cm}$. The volume of liquid of each of the 21 layers was increased so that the free surface was level with the top of the sidewalls. A raised cover was set on top of the free surface to reduce evaporation. All other procedures remained the same.

\section{Results and discussion}

A total of 27 experiments were conducted, 17 with salt-water solution and 10 with ethanol-water solution. Out of the total, five experiments with salt water and three with ethanol-water were preliminary in nature. The purpose was to find a suitable combination of the type and amount of tracer particles to use for visualization. Listings of the conditions under which each experiment was conducted are presented in tables 1 and 2.

\subsection{Impulsively applied $\Delta T$}

When a temperature difference larger than the critical value is applied impulsively across a tank, a vertical array of convection cells is generated almost immediately along the heated and cooled walls (Chen et al. 1971). This is illustrated in figure 1 by a series of three shadowgraphs taken at the start of Experiment 26 with an ethanol-water solution. A $\Delta T$ of $9{ }^{\circ} \mathrm{C}$ was imposed impulsively by pre-heating and -cooling the fluids in the two constant-temperature baths and then opening the valves simultaneously to allow the fluids to circulate in their respective sidewalls. The response of the tank is quite rapid: $\Delta T$ reached $8.7^{\circ} \mathrm{C}$ within 2 min. Figure $1(a)$ shows two convection cells starting at the lower right corner adjacent to the heated wall and one cell at the upper left corner adjacent to the cold wall at $1 \mathrm{~min} .20 \mathrm{~s}$ after the start of the experiment. The early onset of convection at these locations is due to the reduced solute gradient resulting from the non-diffusive boundary conditions at the upper and lower boundaries. At $t=4 \mathrm{~min}$, figure $1(b)$, arrays of convection cells had started from both the hot and cold walls. The bottom cell is seen to advance rapidly toward the cold wall. By $t=6$ min., figure $1(c)$, the bottom cell had reached about halfway across the tank, and all other cells had similarly grown sideways. Eventually, the cells spanned the entire width of the tank.

The flow pattern in each of the convection cells consisted of a flow of relatively cold, 
(a)

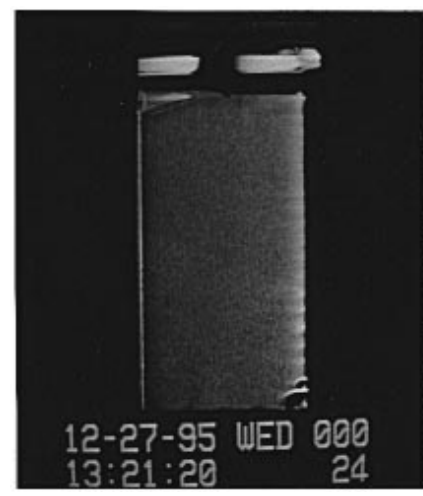

(b)

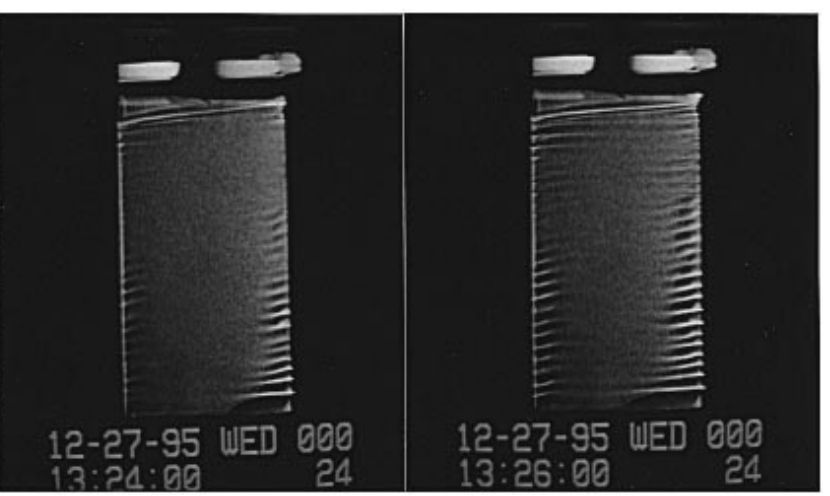

FIGURE 1. Growth of convection cells due to a $\Delta T=9^{\circ} \mathrm{C}$ applied at $t=13: 20$. Experiment 26, ethanol-water solution with a rigid top boundary. Hot wall on the right. (a) $t=13: 21 ;(b) t=13: 24$; (c) $t=13: 26$.

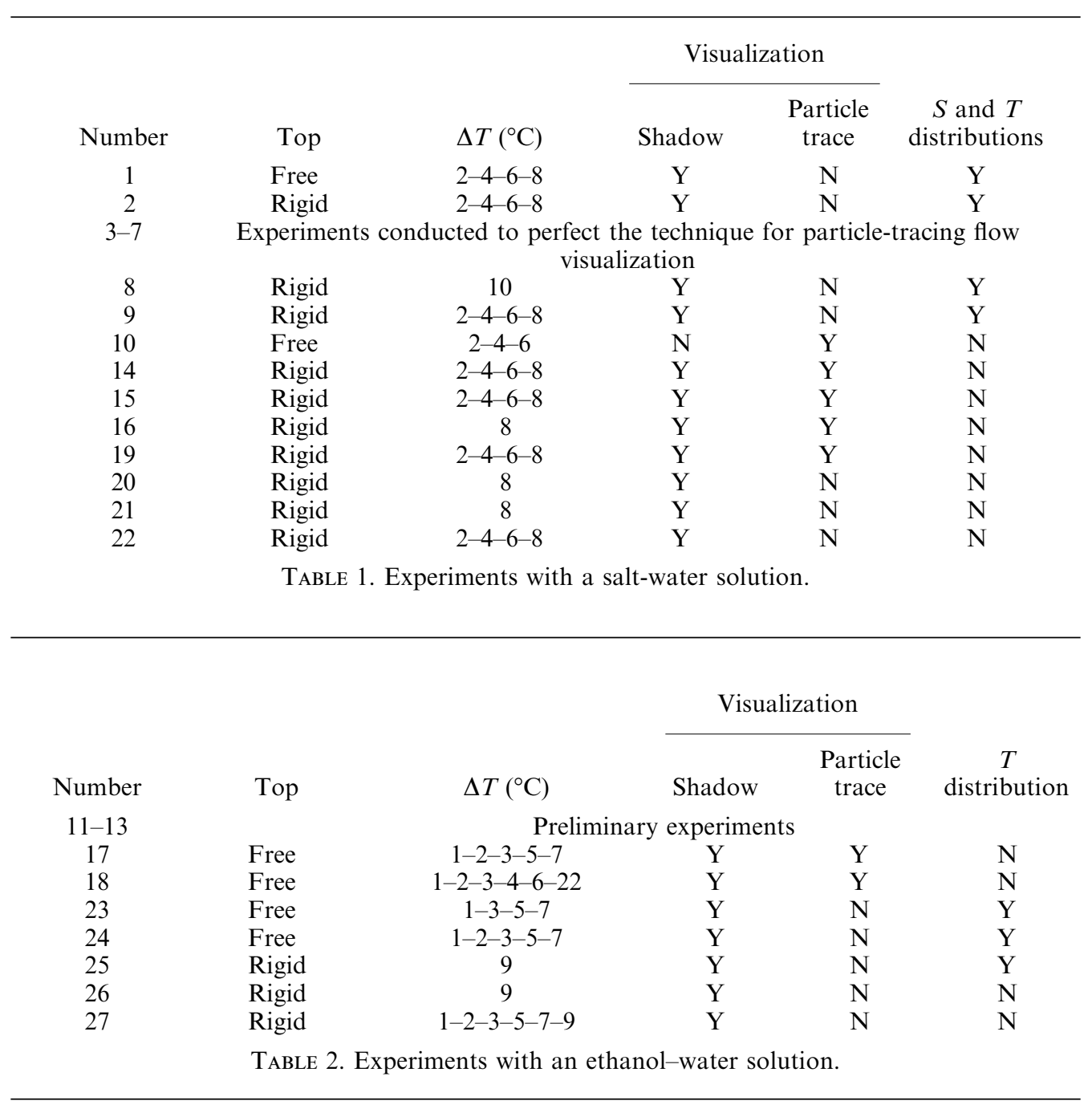


(a)

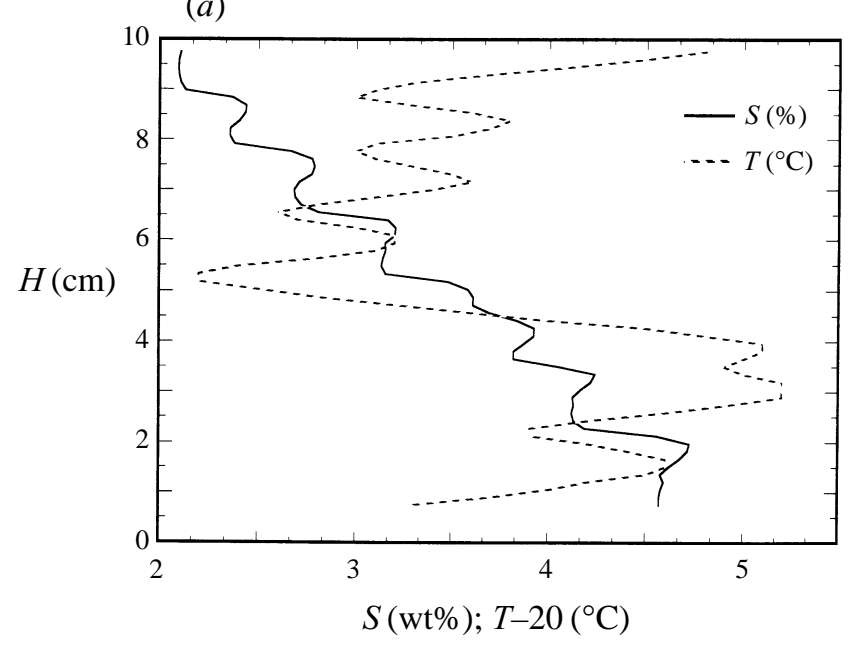

(b)

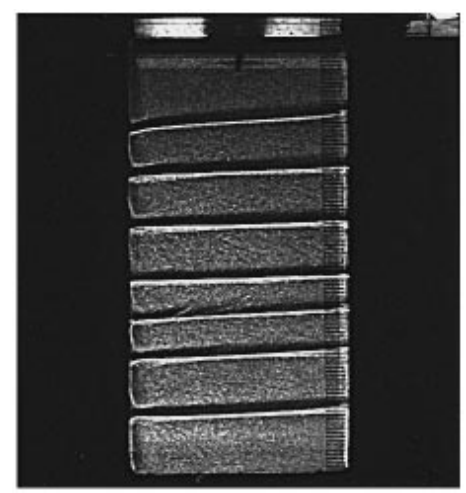

FIGURE 2. Temperature and solute concentration distributions along the vertical centreline of the tank. Experiment 8, with salt-water solution with a rigid top. Data taken at $39 \mathrm{~min}$. after an impulsively applied $\Delta T=10^{\circ} \mathrm{C}$. (a) $T$ and $S$ distributions, the vertical position of the probes was shifted $3 \mathrm{~mm}$ upward; (b) shadowgraph showing the convection cells.

solute-rich fluid along the bottom of the cell toward the hot wall. It then rose along the hot wall due to heating. After reaching the height where the fluid becomes neutrally buoyant, the stream turned inward and flowed toward the cold wall. The relatively warmer and solute-rich fluid was then being brought on top of cooler and solute-poor fluid, a situation in which salt fingers are likely to occur.

We made simultaneous measurements of the temperature, $T$, and concentration, $C$, in a similar salt-solution (Experiment 8), which are shown in figure 2(a), with a shadowgraph of the convection cell prsented in figure $2(b)$ for reference. A $\Delta T$ of $10^{\circ} \mathrm{C}$ was impulsively imposed initially. The data were taken at $t=39 \mathrm{~min}$., with readings at every $2.5 \mathrm{~mm}$. In this and subsequent similar figures (4 and 8), continuous distributions of $S$ and $T$ were obtained by connecting the data points by straight lines. The abscissa is in wt \% and in $\left(T-20^{\circ} \mathrm{C}\right)$ in order to exhibit both curves on the same graph. The shadowgraph was taken 1 min. later. At that time, there were eight convection cells. With the exception of the topmost cell and the fifth one from the top, both temperature and concentration increased upward in all the cells. Using the data for $T$ and $C$ of the 
bottom cell at $0.3 \mathrm{~cm}$ and $1.2 \mathrm{~cm}$ from the bottom, where the temperature was maximum, the values of the thermal and solute Rayleigh number, $R$ and $R_{S}$, are

$$
R=\frac{g \alpha \Delta T d^{3}}{\kappa \nu}=1.96 \times 10^{4}, \quad R_{S}=\frac{g \beta \Delta C d^{3}}{\kappa_{S} \nu}=1.82 \times 10^{6},
$$

with $d=0.9 \mathrm{~cm}$ and the following thermal physical properties: $\alpha=3.0 \times 10^{-4} \mathrm{C}^{-1}$, $\beta=7.2 \times 10^{-3}(\mathrm{wt} \%)^{-1}, \kappa=1.42 \times 10^{-2} \mathrm{~cm}^{2} \mathrm{~s}^{-1}$, and $\kappa_{S}=1.56 \times 10^{-5} \mathrm{~cm}^{2} \mathrm{~s}^{-1}$. The value of $\kappa_{S}$ is from Rard \& Miller (1979). It is to be noted that $\alpha \Delta S$ and $\beta \Delta S$ are equal in magnitude in this case.

For a free-free layer, the stability criterion for onset of salt-finger convection is (Stern 1960)

$$
R_{S}>R+\frac{27}{4} \pi^{4} .
$$

For the bottom layer, the lower boundary is rigid and the constant in (4) should be replaced by $\sim 10^{3}$. In this case, since $R_{S} \gg R>10^{3}$, the layer is in a supercritical state regardless of the boundary conditions. It is reasonable to expect that, in most of the rest of the convection cells, similar supercritical conditions exist. Since strong shear is present in each convection cell, finger convection will appear as two-dimensional rolls aligned in the direction of shear, as shown by Linden (1974) in counterflowing streams of sugar and salt solution.

In fact, our particle trace photos, presented in figure 3, show that salt-finger convection occurs as soon as convection cells are generated along the hot wall. The first five photos were taken during the first $6 \mathrm{~min}$. of Experiment 16 using the salt solution. A $\Delta T$ of $7.3^{\circ} \mathrm{C}$ was imposed impulsively at $10: 25$. The laser sheet was at $0.5 \mathrm{~cm}$ above the bottom boundary. Two minutes later, figure 3(a), salt fingers can be seen to advance from the hot wall (top) toward the cold wall. The finger convection sheets are more distinct at 10:28, figure $3(b)$, and are quite similar in appearance to those in the shadowgraphs of Linden (1974) and schlieren pictures of Thangam \& Chen (1981). The bright regions indicate regions of little or no vertical motion, and the dark regions indicate ascending or descending flow. At $4 \mathrm{~min}$. into the experiment, figure $3(\mathrm{c})$, the fingers had advanced with a uniform front. One minute later, however, the front is rather uneven, as shown in figure $3(d)$. At 10:31, figure 3(e), the fingers seem to have reached the cold wall and become quite well organized, again. Figure $3(f)$ shows the fingers at $25 \mathrm{~min}$. into the experiment. They persisted throughout the duration of the experiment, 4-5 h. Of course, eventually, the fingers would disappear when the fluid was well mixed. Similar onset processes of finger convection appeared in all other cells except the topmost one. These processes took a longer time to establish than that for the bottom cell. Eventually, regular finger structures appeared in all the cells.

It has been suggested that the three-dimensional structure observed here may be caused by Rayleigh-Taylor instability in the presence of shear. This is not supported by experimental evidence. Snider \& Andrews (1994) carried out such experiments and observed that the flow initially forms large spanwise vortices that break up into threedimensional buoyancy plumes downstream. They do not appear as longitudinal rolls.

\subsection{Gradual increase of $\Delta T$}

In this series of experiments, $\Delta T$ across the tank was increased gradually, usually in $2{ }^{\circ} \mathrm{C}$ steps. Temperature adjustments were made at $1 \mathrm{hr}$ intervals, approximately 0.8 diffusion time across the tank. For the experimental salinity gradient and test-tank width, the critical $\Delta T$ is $6.5^{\circ} \mathrm{C}$ (Thorpe et al. 1969). In one such experiment, Experiment 9, temperature and concentration surveys were made approximately 
(a)

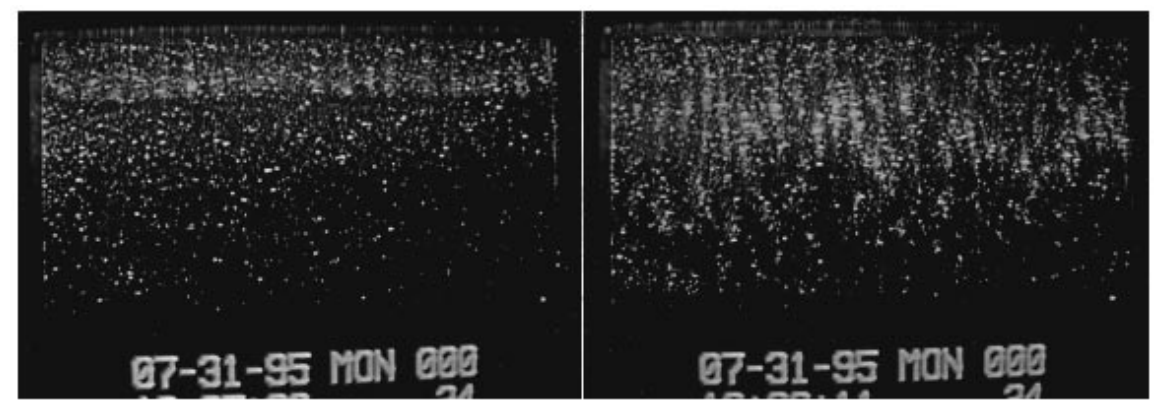

(b)

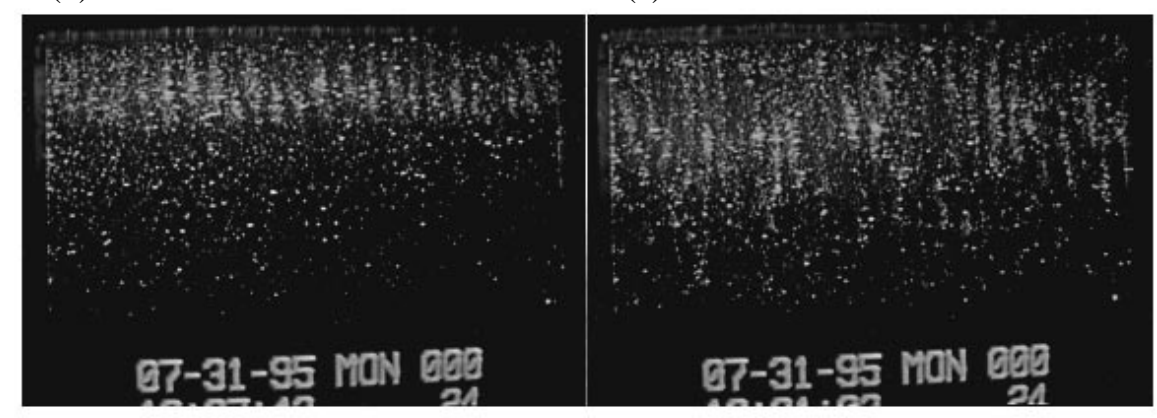

(c)

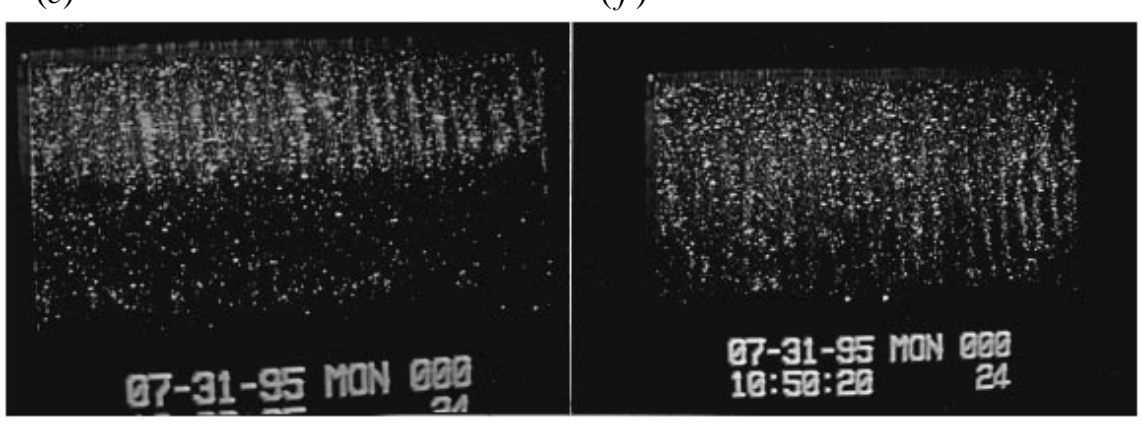

FIGURE 3. Particle trace photos showing the onset of salt fingers as soon as convection is initiated by impulsively applying a $\Delta T=7.3{ }^{\circ} \mathrm{C}$ at $t=10: 25$. Experiment 16 , salt solution with a rigid top. Laser sheet at $0.5 \mathrm{~cm}$ above the bottom boundary. (a) $t=10: 27 ;$ (b) $t=10: 28 ;$ (c) $t=10: 29 ;(d)$ $t=10: 30 ;(e) t=10: 31 ;(f) t=10: 50$.

10 min. prior to every $\Delta T$ adjustment. The first four sets of measurements are shown in figure 4. Figure 4(a) shows the initial temperature and salinity distribution in the tank prior to heating. The salt concentration was nearly linear, and there was a slight temperature anomaly at the top. We note here that the temperatures were recorded in $0.1{ }^{\circ} \mathrm{C}$ increments. The stepwise changes in the temperature distributions were due to instrument limitations rather than actual discontinuities. The first $\Delta T$ adjustment was made at $t=10: 10$. At $t=11: 00$, when the $\Delta T$ was $2.1^{\circ} \mathrm{C}$, the temperature became a little more stratified and there was hardly any change in the concentration distribution, as shown in figure $4(b)$. At this time, there was no motion in the tank, as indicated by the shadowgraph. At $t=12: 00, \Delta T=3.8^{\circ} \mathrm{C}$, figure $4(c)$, there was a strong convecting 

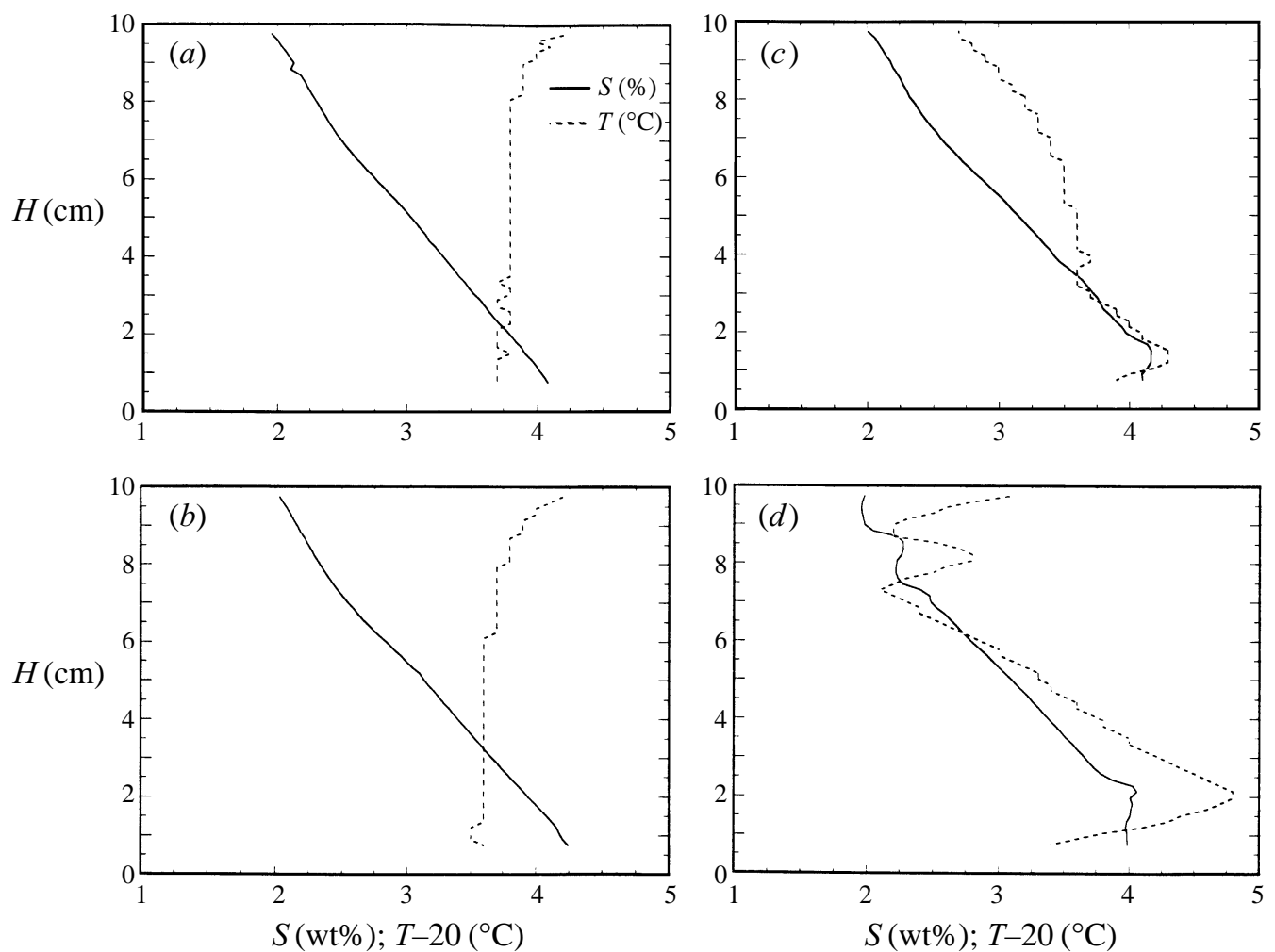

FIGURE 4. Evolution of the vertical distributions of temperature and concentration as $\Delta T$ is gradually increased. Experiment 9, salt solution with a rigid top. The first $\Delta T$ step was applied at $T=10: 10$. (a) $t=10: 00, \Delta T=0{ }^{\circ} \mathrm{C}$; (b) $t=11: 00, \Delta T=2.1{ }^{\circ} \mathrm{C}$; (c) $t=12: 00, \Delta T=3.8^{\circ} \mathrm{C}$; (d) $t=13: 05$, $\Delta T=5.4^{\circ} \mathrm{C}$.

layer at the bottom distorting both the temperature and concentration distributions. Above this layer, the concentration stratification was not affected; however, the temperature distribution assumed a negative gradient due to the high temperature at the top of the convecting layer near the bottom boundary. The shadowgraph shows a strong layer at the bottom, but, at the top, there was a small convection cell at the cold wall, approximately $0.5 \mathrm{~cm}$ thick, extending approximately $1 \mathrm{~cm}$ into the tank. The rest of the fluid remained quiescent. At $13: 05$ with $\Delta T=5.4^{\circ} \mathrm{C}$, figure $4(d)$, two convecting layers were established near the top, $1.5 \mathrm{~cm}$ and $1.0 \mathrm{~cm}$ in thickness, and a strong convecting layer near the bottom with a thickness of $1.5 \mathrm{~cm}$. Within the middle $5.5 \mathrm{~cm}$ section of the tank, a stable salinity gradient evolved from the initial salinity distribution was still being maintained. The temperature distribution showed a negative constant gradient. This is due to the presence of warmer fluid along the top of the bottom convection layer and the cooler fluid along the bottom of the top convection layer. In the midsection of the tank, the stabilizing effect of the salinity gradient $\left(R_{S} \sim-6.4 \times 10^{9}\right)$ far exceeded the destabilizing effect of the adverse temperature gradient $\left(R \sim-4 \times 10^{6}\right)$. Diffusive instability due to heating from below of a salinity gradient could not occur, and the fluid remained quiescent. Further increases in $\Delta T$ across the tank would bring the fluid in the midsection into the unstable regime with respect to sideways heating, and horizontal convection layers would be generated. Onset of these layers was generally through a series of vortices, and we illustrate this phenomenon with a series of shadowgraphs. 


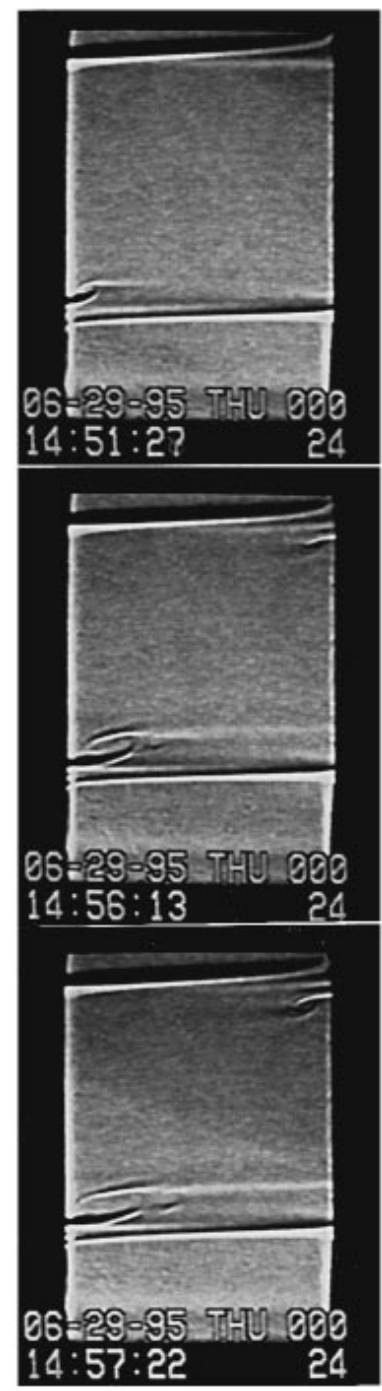

(a)

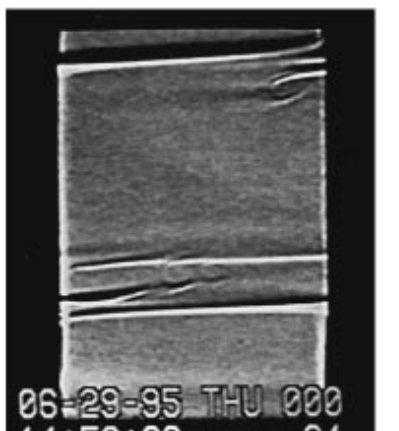

(b)

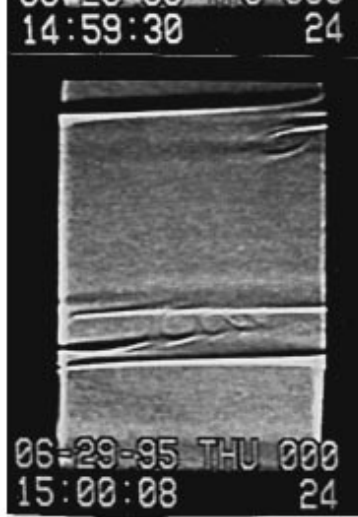

$(c)$

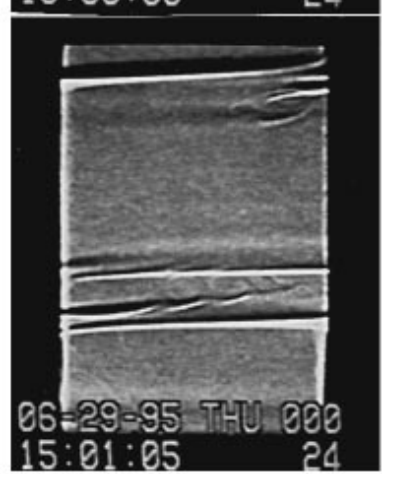

$(d)$

(e)

$(f)$

FIGURE 5. Shadowgraph showing the successive generation of vortices. Experiment 2, salt solution with a rigid top. $\Delta T$ was gradually increased to $8^{\circ} \mathrm{C}$ at $t=14: 46$, exceeding the critical value of $6.5^{\circ} \mathrm{C}$. (a) $t=14: 51$, starting vortex at the cold wall (left); (b) $t=14: 56$, two vortices; (c) $t=14: 57$; (d) $t=14: 59$, three vortices; (e) $t=15: 00$, five vortices; $(f) t=15: 01$, vortices fading.

These shadowgraphs were obtained in a similar experiment, Experiment 2. This was started with an initial $\Delta T$ of $2{ }^{\circ} \mathrm{C}$ at $11: 45$, and $\Delta T$ was increased in $2{ }^{\circ} \mathrm{C}$ increments every hour. At $14: 30$, when the $\Delta T$ was $6{ }^{\circ} \mathrm{C}$, the top layer grew to $2.3 \mathrm{~cm}$ thick and the bottom layer to $1.8 \mathrm{~cm}$ thick while the fluid in the mid-tank remained quiescent. At $14: 46, \Delta T$ was increased to $8^{\circ} \mathrm{C}$, exceeding the critical value of $6.5^{\circ} \mathrm{C}$. The series of shadowgraphs shown in figure 5 were taken in a $10 \mathrm{~min}$. interval from 14:51 to 15:01.

At 14:51, there was a small starting vortex with counterclockwise rotation along the cold wall just above the bottom convecting layer, as shown in figure 5(a). Five minutes later, figure $5(b)$, it became more intensified and a secondary vortex, rotating in the same direction, was induced right next to the first one. We note here that the top portion of the tank, approximately $2 \mathrm{~cm}$ in thickness, was outside the field of view when the camera was zoomed in for an enlarged view. At 14:57, figure 5(c), the second 
vortex grew in size and, at 14:59, figure $5(d)$, a third vortex was generated. By this time, the deficiencies in the salt concentration between the present layer and the rest of the fluid above it became large enough to generate a rather distinct boundary in the shadowgraph. At 15:00, figure 5(e), five counterclockwise vortices can be seen spanning from the cold to the hot wall. By 15:01, figure $5(f)$, the vortices began to fade due to mixing.

The onset of the second layer was at the cold wall rather than at the hot wall. The physical mechanism is as follows. Consider the fluid adjacent to the cold wall just above the bottom layer. As the temperature of the cold wall is adjusted downward, a less concentrated fluid parcel is brought downward by the negative buoyancy. Upon meeting the upper boundary of the convecting layer, it turns into the tank and flows above the warm current coming from the hot wall. The negative buoyancy of the fluid parcel is removed by heating, and it flows upward and completes the vortex flow. The first vortex, being cooled by the cold wall, in turn, cools the neighbouring fluid and causes the onset of the next vortex. This chain of events cannot be initiated in the fluid above the bottom convecting layer adjacent to the hot wall. However, for the fluid adjacent to the hot wall just below the top convecting layer, the same mechanism will initiate a series of vortices. The first of such vortices can be seen in figure $5(c-e)$.

The onset of convection layers through successive vortex generation when $\Delta T$ was gradually increased was also recorded in particle trace photographs. These were taken during Experiment 15 with the salt solution, and they show that salt fingers are generated as soon as the first vortex flow is established. The experiment was started at $11: 43$, with an initial $\Delta T$ of $2.5^{\circ} \mathrm{C}$. The $\Delta T$ was increased to $4.5^{\circ} \mathrm{C}$ at $12: 43$ and to $6.9^{\circ} \mathrm{C}$ at $13: 43$. At this time, the bottom layer was $1.8 \mathrm{~cm}$ thick at the hot wall and $1.6 \mathrm{~cm}$ at the cold wall. The horizontal laser sheet was at $2.2 \mathrm{~cm}$ above the bottom boundary. At 13:51, figure 6(a), the presence of a starting vortex can be discerned as a band of alternating bright and dark regions near the bottom of the photo which was the cold wall. At 13:55, figure 6(b), the first vortex intensified with indications of the onset of finger convection, and a second vortex was starting. At 13:57, figure 6(c), finger convection was fully developed in the first vortex and the second vortex was intensified. By 14:00, figure $6(d)$, a third vortex was clearly visible, and it became intensified at 14:02, figure 6(e). By 14:06, figure $6(f)$, the vortices spanned the entire width of the tank, and finger convection could be seen in the third vortex.

\subsection{Finger convection generated by surface-tension-driven flow}

In all the experiments discussed so far, the flow was generated by buoyancy. In both the impulsively imposed $\Delta T$ and the gradually increased $\Delta T$ cases, the top convecting layer always had a nearly uniform or stable concentration distribution and a stable temperature distribution, as shown in figures 2 and 4 . No evidence of salt fingers was ever detected by the particle trace method, although particles were seen to move across the tank due to convection. However, if the top surface of the layer is free, a thermocapillary flow will be generated as soon as a $\Delta T$ is imposed. It is likely that such a flow can create temperature and concentration distributions conducive to the onset of finger convection. Our experimental results indicate that such is the case.

The experiments were done with ethanol, a fluid whose surface is not easily contaminated. The density stratification was achieved by using $100 \%$ ethanol for the surface layer and a $64 \%$ ethanol-water solution at the bottom layer. A raised cover with an air gap of $\sim 2 \mathrm{~mm}$ was placed on top of the tank to minimize evaporation. During the $1 \mathrm{~h}$ wait after filling, the particles near the free surface remained stationary after the initial motion due to filling had ceased. This indicates that there was no 
(a)

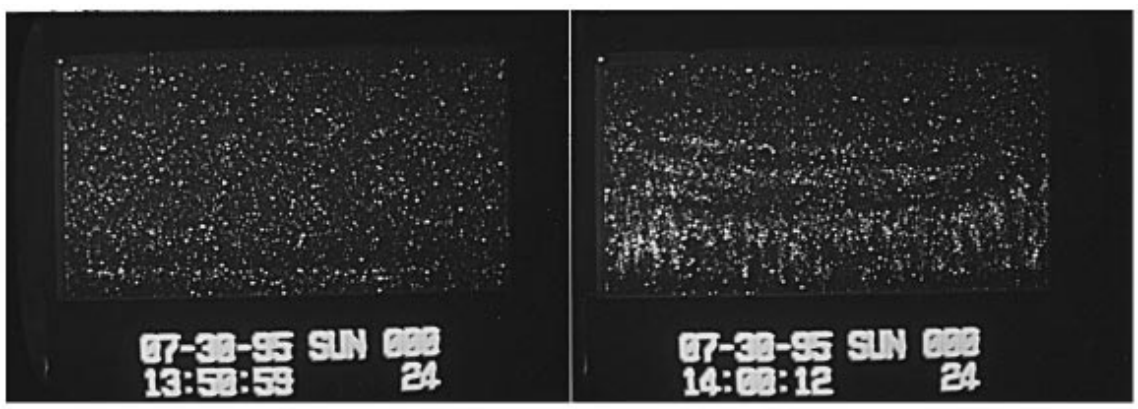

(b)

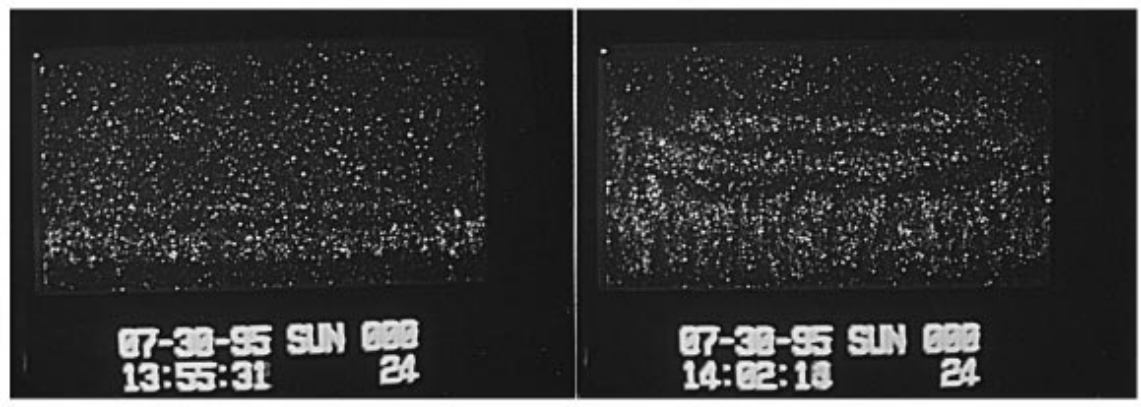

(c)

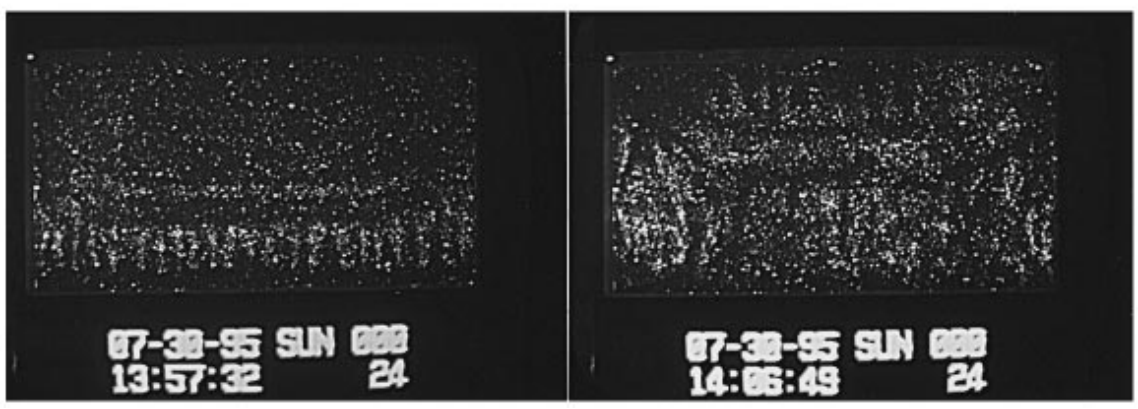

Figure 6. Particle trace photos for the case of gradually increased $\Delta T$. Experiment 15 , salt-water solution with a rigid top. $\Delta T$ was increased to $6.9^{\circ} \mathrm{C}$ at $t=13: 43$. (a) $t=13: 51$, starting vortex; (b) $t=13: 55$, starting of the second vortex; (c) $t=13: 57 ;(d) t=14: 00$, third vortex; (e) $t=14: 02$; (f) $t=14: 06$.

evaporation-induced motion on the free surface. In Experiment 18, the first $\Delta T$ step of $0.8^{\circ} \mathrm{C}$ was imposed at $10: 10$. Particles at a level $0.5 \mathrm{~cm}$ below the free surface were seen to move slowly from the hot wall toward the cold wall soon after the $\Delta T$ was imposed. Figure $7(a)$ shows a particle trace photograph taken at 10:14 with an $8 \mathrm{~s}$ exposure time. Motion of the particles can be discerned, and the speed of the particles is higher toward the cold wall (bottom of photo). There were no apparent salt fingers. At 10:18, figure $7(b)$, finger convection was clearly exhibited near the cold wall. At 11:55, these fingers became better organized at a higher $\Delta T$ of $1.9^{\circ} \mathrm{C}$, figure $7(c)$. At $15: 14$, when $\Delta T$ was increased to $5.8^{\circ} \mathrm{C}$, finger convection still persisted.

The reason that the flow is more vigorous and the salt fingers first appear near the cold wall is due to the effect of water concentration on the surface tension of an 


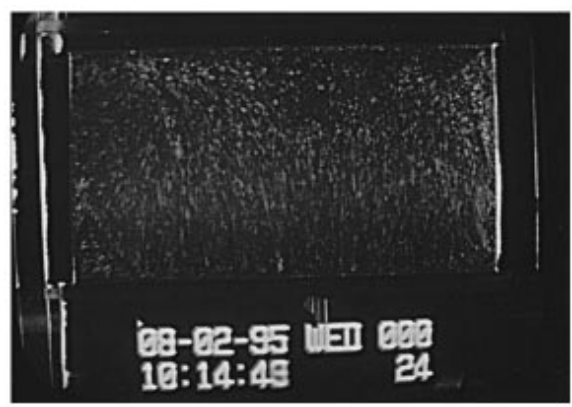

(a)

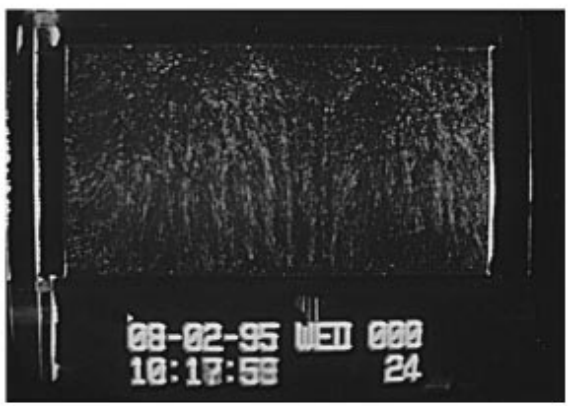

(b)

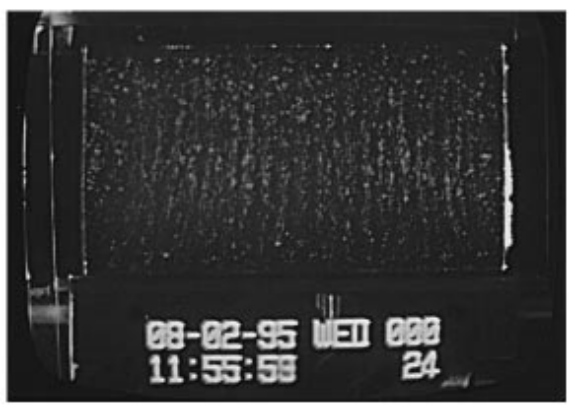

(c)

FIGURE 7. Finger generation by surface-tension-driven flows. Experiment 18, ethanol-water solution with a top free surface. $\Delta T=0.8^{\circ} \mathrm{C}$ was imposed at $t=10: 10$ and the laser sheet at $0.5 \mathrm{~cm}$ below the free surface. (a) $t=10: 14$, motion but with no salt fingers; (b) $t=10: 18$, salt fingers can be seen near the cold wall; (c) $t=11: 55, \Delta T=1.9^{\circ} \mathrm{C}$ set at $11: 10$, regular salt-finger array.

ethanol-water solution. Surface tension of such a solution increases with increasing concentrations of water. (The surface tension of water is approximately three times that of ethanol.) As a $\Delta T$ is imposed, thermocapillary motion will induce two vortices, one each at the hot and cold walls. The vortex at the hot wall tends to increase the water concentration of the surface fluid, thus increasing the surface tension and partly nullifying the capillary effect. The opposite is true for the vortex flow near the cold wall. There, the increased concentration of water will enhance the capillary effect and produce a more vigorous flow, causing the early appearace of salt fingers.

Temperature distributions in the fluid for an experiment with a free surface (Experiment 24) and with a rigid top (Experiment 27) are shown in figure 8. The initial temperature of the fluid in Experiment 24 was $19.5^{\circ} \mathrm{C}$ and that for Experiment 27 was $22.1^{\circ} \mathrm{C}$; data were taken at $\Delta T=1{ }^{\circ} \mathrm{C}, 2{ }^{\circ} \mathrm{C}$, and $3{ }^{\circ} \mathrm{C}$. The temperature distribution in Experiment 27 (rigid top surface) was similar to those obtained in salt-solution Experiment 9 shown in figure 4. This indicates that fluid in the layer was still stably 


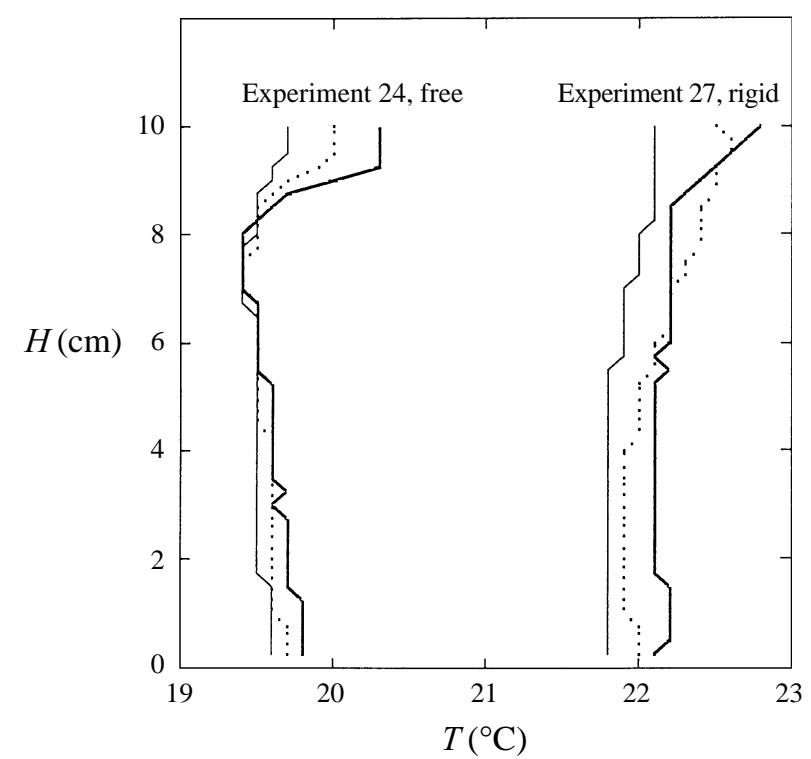

FIGURE 8. Temperature distribution for an experiment with a free surface (Experiment 24) and with a rigid surface (Experiment 27). $-\Delta T=1{ }^{\circ} \mathrm{C} ; \cdots \cdots, \Delta T=2{ }^{\circ} \mathrm{C} ;-, \Delta T=3{ }^{\circ} \mathrm{C}$.

stratified and motionless. This was confirmed by the particle trace visualization. The data obtained for the free-surface case, Experiment 24, indicated that there was motion, even at $\Delta T=1{ }^{\circ} \mathrm{C}$. The top $0.5 \mathrm{~cm}$ was well mixed due to thermocapillary motion. As $\Delta T$ was increased, the motion extended further downward. It is conjectured that the concentration distribution in the top layer was unstable, thus causing the onset of finger convection. Even at $\Delta T=3{ }^{\circ} \mathrm{C}$, for both the free-surface and rigid-surface cases, the temperature distribution in the rest of the tank was essentially undisturbed, except near the bottom, where there was convection in the bottom layer.

\section{Conclusions}

When a fluid layer stably stratified by solute concentrations is subjected to a lateral temperature difference, horizontal convection cells are generated. Within each of these cells, warm solute-rich fluid is brought above the cooler solute-poor fluid by convection motion, causing the onset of finger convection. In the case of suddenly imposed $\Delta T$, convection cells advance from the hot wall toward the cold, and finger convection is immediately generated as the convection cells are formed. The fingers then advance with the convection cells toward the cold wall. For a gradually increased $\Delta T$, when the critical $\Delta T$ is exceeded the convection layers are generated successively. Because the fluid is at the marginal state, the convection layer is formed quickly by a series of vortices originating at the wall. Finger convection is again generated immediately and advances toward the opposite wall in waves. Once fully developed, the fingers persist within the convection layer until the solute concentration becomes uniform. These results show that all two-dimensional numerical simulations, including our early work (Wirtz et al. 1972), were based on a physically unrealistic flow model. Therefore, the vertical transport of solute and heat would be grossly underpredicted.

When the top surface is rigid, the topmost layer consists of nearly uniformconcentration fluid with a stable temperature gradient. Convective motion within the layer is without fingers. When the top surface is free, thermocapillary motion is 
generated as soon as a small $\Delta T$ is imposed. The initial $\Delta T$ steps in these experiments were small so that there was no buoyancy-generated motion. Thermocapillary motion brings the warmer and solute-rich fluid to the surface and causes the onset of finger convection. Since the presence of solute increases the surface tension, the initial thermocapillary effect is enhanced (retarded) at the cold (hot) wall. As a result, finger convection starts at the cold wall.

Because of the small magnitude of the mass diffusivity of materials, salt fingers can be generated at much reduced gravity levels. Using the thermophysical properties of a liquid lead-tin alloy given by Coriell, Cordes \& Boettinger (1980), in a $1 \mathrm{~cm}$ thick layer with a concentration difference of $1 \mathrm{wt} \%$ across the layer, salt fingers can appear at $10^{-5} g_{0}$, where $g_{0}$ is the gravitational acceleration at sea level. In such a low-gravity environment, motion driven by a surface tension gradient can be readily generated by a temperature gradient. Such flows may be used to promote mixing in a fluid layer with stratified mass concentration.

Funding of this research by NASA through Microgravity Science and Application Grant NAG3-1328 and by the Republic of China, National Science Council Grant 852212-E-002-010, is gratefully acknowledged. We are indebted to Dr Paul Kolodner, who shared with us his extensive knowledge of the thermal physical properties of ethanol-water solutions. We also thank Dr Cho Lik Chan for his stimulating discussions on the surface tension case.

\section{REFERENCES}

Chen, C. F., Briggs, D. G. \& Wirtz, R. A. 1971 Stability of thermal convection in a salinity gradient due to lateral heating. Intl J. Heat Mass Transfer 14, 57-65.

Chen, Y.-M. \& Liou, J.-K. 1997 Time-dependent double-diffusive convection due to salt stratified fluid layer with differential heating in an inclined cavity. Intl J. Heat Mass Transfer 40, 711-725.

Coriell, S. R., Cordes, M. R. \& Boettinger, W. J. 1980 Convective and interfacial instabilities during unidirectional solidification of a binary alloy. J. Cryst. Growth 49, 13-28.

Hart, J. E. 1971 On sideways diffusive instability. J. Fluid Mech. 49, 279-288.

Hart, J. E. 1973 Finite amplitude sideways diffusive convection. J. Fluid Mech. 59, 47-64.

HeAD, M. J. 1983 The use of miniature four-electrode conductivity probes for high resolution measurement of turbulent density or temperature variations in salt-stratified water flows. PhD thesis, University of California, San Diego.

HeInRICH, J. C. 1984 A finite element model for double-diffusive convection. Intl J. Numer. Meth. Engng 20, 447-464.

Huppert, H. E., Kerr, R. C. \& Hallworth, M. A. 1984 Heating or cooling a stable compositional gradient from the side. Intl J. Heat Mass Transfer 27, 1395-1401.

Huppert, H. E. \& Turner, J. S. 1980 Ice blocks melting into a salinity gradient. J. Fluid Mech. 100, $367-384$.

Kerr, O. S. 1989 Heating a salinity gradient from a vertical sidewall: linear theory. J. Fluid Mech. 207, 323-352.

Kerr, O. S. 1990 Heating a salinity gradient from a vertical sidewall: nonlinear theory. J. Fluid Mech. 217, 529-546.

KnOBloch, E. 1980 Convection in binary fluids. Phys. Fluids 23, 1918-1920.

LAndolt, H. \& Bornstein, R. 1989 Eigenschaften der Materie in ihren aggregatzustanden. 5.Teil., p. 640, Springer.

Lee, J. W. \& Hyun, M. T. 1991 Time-dependent double diffusion in a stably stratified fluid under lateral heating. Intl J. Mass Heat Transfer 34, 2409-2415.

Linden, P. F. 1974 Salt fingers in a steady shear flow. Geophys. Fluid Dyn. 6, 1-27.

Rard, J. A. \& Miller, D. G. 1979 The mutual diffusion coefficients of $\mathrm{NaCl}-\mathrm{H}_{2} \mathrm{O}$ and $\mathrm{CaCl}_{2}-\mathrm{H}_{2} \mathrm{O}$ at $25^{\circ} \mathrm{C}$ from Rayleigh interferometry. J. Solution Chem. 8, 701-716. 
SNIDER, D. M. \& ANDrews, M. J. 1994 Rayleigh-Taylor and shear driven mixing with an unstable thermal stratification. Phys. Fluids 6, 3324-3334.

Stern, M. E. 1960 The 'salt-fountain' and thermohaline convection. Tellus 12, 172-175.

TANnY, J. \& TsinOBER, A. B. 1988 The dynamics and structure of double-diffusive layers in sidewall heating experiments. J. Fluid Mech. 196, 135-156.

Thangam, S. \& CHEN, C. F. 1981 Salt finger convection in the surface discharge of heated saline jets. Geophys. Astrophys. Fluid Dyn. 18, 111-146.

Thangam, S., Zebib, A. \& Chen, C. F. 1982 Double-diffusive convection in an inclined fluid layer. J. Fluid Mech. 116, 363-378.

Thorpe, S. A., Hutt, P. K. \& Soulsby, R. 1969 The effects of horizontal gradients on thermohaline convection. J. Fluid Mech. 38, 375-400.

Wirtz, R. A., Briggs, D. G. \& Chen, C. F. 1972 Physical and numerical experiments on layered convection in a density-stratified fluid. Geophys. Fluid Dyn. 3, 265-288.

Wright, J. \& ShYY, W. 1996 Numerical simulation of unsteady convective instrusions in a thermohaline stratification. Intl J. Mass Heat Transfer 39, 1183-1201. 